HYBRID NANOSTRUCTURES

\section{DNA stitched with proteins}

Science 355, eaam5488 (2017)

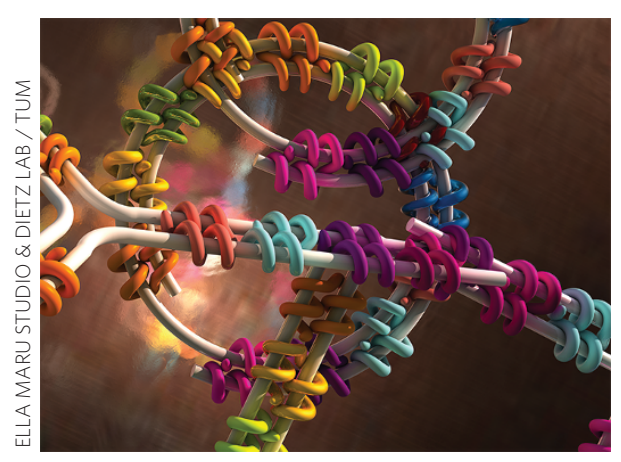

DNA origami can potentially be used to control the spatial arrangement of molecules in living systems, but cells are challenging environments for DNA origami, due to the lack of single-stranded DNA and the need for a high annealing temperature. Praetorius and Dietz now report a way of folding double-stranded DNA templates into defined nanostructures in vitro, a first step towards in vivo conditions. Instead of using oligonucleotide staples, common in DNA origami, two parallel DNA double strands are stitched together by staple proteins originated from transcription activator-like (TAL) effector proteins.

Praetorius and Dietz synthesize twelve different staple proteins by programming the genes that encode TAL effectors - each staple protein can recognize a specific DNA sequence. By designing the sequences of the template DNA using a polymerase chain reaction, the scientists fabricate various desired hybrid structures at room temperature. Moreover, the synthesis can be carried out in one pot containing the genes encoding the staple proteins, RNA polymerase, ribosomes, cofactors for transcription and translation, and the double-stranded DNA scaffold. The encoded staple proteins self-assemble with the parallel DNA double strand, forming the desired DNA-protein-hybrid nanostructure. With this method, proteins with different functionalities can also be easily integrated into hybrid nanostructures and their spatial arrangement can be accurately controlled.

\section{SELF-ASSEMBLY}

\section{Block molecules separate}

ACS Nano http://doi.org/b5m6 (2017)

Liquid crystals and block co-polymers are characterized by a morphological order that results from interactions at the molecular level. Whereas in block co-polymers the incompatibility between the blocks causes a phase separation that minimizes the interfacial energy, in liquid crystals intermolecular interactions are mediated by molecular orientation. Now, Berrocal et al. have studied an intermediate regime in which molecules composed of incompatible blocks of naphthalenediimides (hard block) and oligodimethylsiloxanes (soft block) assemble in defined morphologies.

The researchers study two different families of compounds. In the first one, either one or two naphthalenediimide groups are end-capped by dimethylsiloxanes of various lengths. In the second one, the naphthalenediimide groups terminate the dimethylsiloxane oligomer. The first family self-assembly behaviour is driven by the $\pi-\pi$ stacking of the hard moiety forming columnar structures in which the intermediate volume is occupied
SENSORS

\section{Gas-sniffing drones}

High levels of air pollution pose a direct threat to global public health. The first step to tackling this problem is to reliably measure and map the concentration of pollutants. To that end, inexpensive portable electronics can come in handy. Fahad et al. have now reported a low-power chip-scale platform for high-sensitivity gas detection.

The gas-sensing chip consists of multiple arrays of chemical-sensitive field-effect transistors with strongly $\mathrm{n}$-doped Si channels 3.5-nm long. The doping enables quick carrier depletion of the channel, resulting in large output modulation and improved sensitivity. The Si channel of each individual transistor can be functionalized with three types of thin (sub-5 nm) electrically insulating layers, each of which is designed to selectively detect $\mathrm{H}_{2}$, $\mathrm{NO}_{2}$ or $\mathrm{H}_{2} \mathrm{~S}$. Upon exposure to one of the target gases or a mixture of them, the threshold voltage shifts, indicating a detection event. Once the gas has been detected, integrated $\mathrm{Au} / \mathrm{Cr}$ microheaters are activated to desorb the gas from the sensor and recover the initial baseline conditions. The detection limit for the $\mathrm{H}_{2} \mathrm{~S}$ sensor is $\sim \mathrm{ppb}$, whereas for $\mathrm{H}_{2}$ it is $\sim 3,000 \mathrm{ppm}$. As a proof-of-concept demonstration, Fahad etal. mounted an $\mathrm{H}_{2}$-sensitive sensor onto a microdrone for real-time gas detection during a pre-programmed flight. by the oligomeric chains. The second family, instead, self-assembles into a lamellar morphology with no evidence of intermolecular interaction between the hard blocks. Remarkably, in both cases, these features are under $10 \mathrm{~nm}$.

Using a number of characterization techniques and theoretical studies, Berrocal et al. show that block-molecule morphologies are not the result of interfacial energy minimization, as in the case of block co-polymers. Rather, they result from the interplay between the tendency of the hard block to crystallize and the thermodynamic incompatibility between the hard and the soft blocks, which, in turn, strongly depends on the orientation of the intermolecular interactions, a feature shared with liquid crystals.

$A M$

\section{NANOELECTRONICS}

\section{A 2D microprocessor}

Nat. Commun. 8, 14948 (2017)

With silicon electronics pushed to its technological limits the search is well underway for the next generation of semiconductors. Two-dimensional transition-metal dichalcogenides, and molybdenum disulfide $\left(\mathrm{MoS}_{2}\right)$ in particular, have been considered as potential successors to silicon. Until now, however, the demonstrations of $\mathrm{MoS}_{2}$-based electronics have been limited to field-effect transistors, inverters and basic integrated circuits. Now, the technological challenge of realizing complex digital circuits based on $\mathrm{MoS}_{2}$ has been partially overcome in a recent report by Wachter and colleagues.

The researchers fabricate a one-bit microprocessor measuring $0.6 \mathrm{~mm}^{2}$ and comprising 115 bottom-gated transistors with a chemical vapour deposition-grown $\mathrm{MoS}_{2}$ bilayer as a channel. Each transistor is characterized by an average field-effect mobility of $3 \mathrm{~cm}^{2} \mathrm{~V}^{-1} \mathrm{~s}^{-1}$ and an on/off ratio of $10^{8}$. Despite the minimal transistor count the circuit contains all essential subunits to perform basic arithmetic and logical operations and can be potentially scaled to multi-bit data. By running an example program, Wachter et al. obtain good signal integrity and rail-to-rail performance of the microprocessor. The feasibility of the presented electronic platform is currently limited by the overall device yield, which remains low due to the non-uniformity of the $\mathrm{MoS}_{2}$ films and other defects introduced during the transfer procedure to prepare the microprocessor.

Written by Olga Bubnova, Alberto Moscatelli and Wenjie Sun. 